\title{
Low-energy antikaon-nuclei interactions studies by AMADEUS: from QCD with strangeness to neutron stars
}

\author{
K. Piscicchia ${ }^{1,2, \star}$, C. Curceanu ${ }^{1}$, M. Cargnelli ${ }^{3}$, R. Del Grande ${ }^{1,4}$, L. Fabbietti ${ }^{5,6}$, J. Marton ${ }^{3}$, \\ A. Scordo ${ }^{1}$, D. Sirghi ${ }^{1}$, I. Tucakovic ${ }^{7}$, O. Vazquez Doce ${ }^{5,6}$, S. Wycech ${ }^{8}$, J. Zmeskal ${ }^{3}$, \\ G. Mandaglio ${ }^{9,10}$, M. Martini ${ }^{1,11}$, and P. Moskal ${ }^{12}$ \\ ${ }^{1}$ INFN, Laboratori Nazionali di Frascati, 00044 Frascati, Italy \\ ${ }^{2}$ Museo Storico della Fisica e Centro Studi e Ricerche Enrico Fermi, Roma, Italy \\ ${ }^{3}$ Stefan-Meyer-Institut für Subatomare Physik, 1090 Wien, Austria \\ ${ }^{4}$ Università degli Studi di Roma Tor Vergata, Rome, Italy \\ ${ }^{5}$ Excellence Cluster 'Origin and Structure of the Universe', 85748 Garching, Germany \\ ${ }^{6}$ Physik Department E12, Technische Universität München, 85748 Garching, Germany \\ ${ }^{7}$ Ruđer Bošković Institute, Zagreb, Croatia \\ ${ }^{8}$ National Centre for Nuclear Research, 00681 Warsaw, Poland \\ ${ }^{9}$ Dipartimento M.I.F.T. dell'Università di Messina, 98166 Messina, Italy \\ ${ }^{10}$ INFN Gruppo collegato di Messina, 98166 Messina, Italy \\ ${ }^{11}$ Dipartimento di Scienze e Tecnologie applicate, Università 'Guglielmo Marconi', 00193 Roma, Italy \\ ${ }^{12}$ Institute of Physics, Jagiellonian University, 30-059 Krakow, Poland
}

\begin{abstract}
The AMADEUS collaboration aims to provide unique quality results from $\mathrm{K}^{-}$ hadronic interactions in light nuclear targets, in order to solve fundamental open questions in the non-perturbative strangeness QCD sector, like the controversial nature of the $\Lambda(1405)$ state, the yield of hyperon formation below threshold, the yield and shape of multi-nucleon $\mathrm{K}^{-}$absorption, processes which are intimately connected to the possible existence of exotic antikaon multi-nucleon clusters and to the role of strangeness in neutron stars. AMADEUS takes advantage of the DAФNE collider, which provides a unique source of monochromatic low-momentum kaons and exploits the KLOE detector as an active target, in order to obtain excellent acceptance and resolution data for $\mathrm{K}^{-}$nuclear capture on $\mathrm{H},{ }^{4} \mathrm{He},{ }^{9} \mathrm{Be}$ and ${ }^{12} \mathrm{C}$, both at-rest and in-flight.
\end{abstract}

\section{Introduction}

The AMADEUS (Anti-kaonic Matter At DAФNE: An Experiment with Unraveling Spectroscopy) [1] experiment investigates the low-energy $\mathrm{K}^{-}$hadronic interaction in light nuclei (e.g. $\mathrm{H},{ }^{4} \mathrm{He},{ }^{9} \mathrm{Be}$ and ${ }^{12} \mathrm{C}$ ) in order to provide experimental constraints on the non-perturbative QCD in the strangeness sector. It uses the low momentum (about $127 \mathrm{MeV} / \mathrm{c}$ ), almost monochromatic, charged kaons provided by the decay of $\phi$ mesons at-rest at the DAФNE factory [2]. The analyses presented here refers to the data acquired by the KLOE [3] collaboration during the 2004/2005 data taking campaign and the data collected by KLOE in collaboration with AMADEUS in the period November-December 2012. The

^e-mail: kristian.piscicchia@1nf.infn.it 
latter data taking was performed by implementing in the central region of the KLOE setup a dedicated pure carbon (graphite) solid target.

In the study of the low-energy QCD with $u, d$ and $s$ quarks the chiral perturbation theory is not applicable, due to the presence of the broad $\Lambda(1405)$ state just few $\mathrm{MeV}$ below the $\overline{\mathrm{K}} \mathrm{N}$ threshold. The $\Lambda(1405)$ is a $J^{P}=1 / 2^{-}$isospin $I=0$ strange baryon resonance, assigned to the lowest $L=1$ supermultiplet of the three-quark system, which decays into $(\Sigma \pi)^{0}$ through the strong interaction. The $\Sigma^{0} \pi^{0}$ decay channel, which is free from the $I=1$ contribution and from the isospin interference term, represents the cleanest signature of the $\Lambda(1405)$ resonance, because not affected by the neighbour $\Sigma(1385)$ resonance. On the other hand, better invariant mass and momentum resolutions are achievable with the reconstruction of the $\Sigma^{+} \pi^{-}$decay channel, which is more sensitive to the resonance shape, once the background is under control. Despite the fact that $\Lambda(1405)$ is a four-stars resonance in Particle Data Group (PDG) [4], its nature still remains an open issue. The three quark picture $(u d s)$ fails to reproduce the observed properties of this state. A review of the theoretical works, and references to the experimental literature can be found in [5]. According to the chiral unitary predictions [6] a high mass pole, coupled to the $\overline{\mathrm{K} N}$ production channel and located around $1420 \mathrm{MeV}$, might contribute to the measured $\Lambda(1405)$ shape. Since the accessible invariant mass, in $\mathrm{K}^{-} \mathrm{p}$ absorption processes, is influenced by the binding energy of the proton in the hosting nucleus, our strategy is to unveil the presence of the high mass pole by exploiting $\mathrm{K}^{-}$captures in-flight $[7,8]$. In this case the kinetic energy of the kaon sets the energy threshold just below the $\overline{\mathrm{K} N}$ threshold. The shape of the $(\Sigma \pi)^{0}$ spectra is also distorted by the non-resonant production below threshold. A key issue, which is addressed in the analyses described below, is the investigation of the non-resonant hyperon-pion transition amplitude below threshold.

The position of the $\Lambda(1405)$ clearly reflects the strength of the $\bar{K} N$ interaction, thus influencing the possible formation of $\overline{\mathrm{K}}$ multi-nucleon bound states. For the di-baryonic kaonic bound state $\mathrm{ppK}^{-}$ theoretical predictions deliver a wide range of binding energies and widths [9], while the experimental results are contradictory [10-16]. Moreover, the extraction of $\mathrm{ppK}^{-}$signal in $\mathrm{K}^{-}$absorption experiments is strongly affected by the yield and the shape of the competing $\mathrm{K}^{-}$double-nucleon absorption (2NA) process.

In Section 2 the features of the DAФNE accelerator and the KLOE detector are summarized. In Section 3 the two collected data samples which were analysed are described. The particle identification procedure is summarised in Section 4. Sections 5 and 6 are dedicated to the obtained results and ongoing analyses, regarding $\mathrm{K}^{-}$multi-nucleon absorption processes, the search for a $\mathrm{ppK}^{-}$signal and the investigation of resonant and non-resonant Hyperon-pion production in light nuclei. The paper ends with conclusions and perspectives.

\section{The KLOE detector at DA $\Phi$ NE}

DAФNE (Double Anular $\Phi$-factory for Nice Experiments) is a double ring $e^{+} e^{-}$collider, designed to work at the center of mass energy of the $\phi$ particle; the $\phi$ meson decay produces charged kaons with low momentum $(\simeq 127 \mathrm{MeV} / \mathrm{c})$ which allows to either stop them, or to explore the products of their low-energy nuclear absorptions.

The KLOE detector is centered around the interaction region of DAФNE and is characterised by an acceptance of $\simeq 98 \%$; it consists of a large cylindrical Drift Chamber (DC) [17] and a fine sampling lead-scintillating fibers calorimeter [18], all immersed in the axially symmetrical magnetic field with intensity of $0.52 \mathrm{~T}$, provided by a superconducting solenoid. The chamber is characterized by excellent position and momentum resolutions. Tracks are reconstructed with a resolution in the transverse $R-\phi$ plane $\sigma_{R \phi} \sim 200 \mu \mathrm{m}$ and a resolution along the $z$-axis $\sigma_{z} \sim 2 \mathrm{~mm}$. The transverse momentum resolution for low momentum tracks $((50<p<300) \mathrm{MeV} / \mathrm{c})$ is $\frac{\sigma_{p_{T}}}{p_{T}} \sim 0.4 \%$. The 
calorimeter is composed of a cylindrical barrel and two endcaps, providing a solid angle coverage of $98 \%$. The volume ratio (lead/fibers/glue $=42: 48: 10$ ) is optimized for a high light yield and a high efficiency for photons in the range (20-300) MeV/c. The photon detection efficiency is $99 \%$ for energies larger than $80 \mathrm{MeV}$ and it falls to $80 \%$ at $20 \mathrm{MeV}$ due to the cutoff introduced by the ADC and TDC thresholds. The position of the clusters along the fibers can be obtained with a resolution $\sigma_{\|} \sim 1.4 \mathrm{~cm} / \sqrt{E(\mathrm{GeV})}$. The resolution in the orthogonal direction is $\sigma_{\perp} \sim 1.3 \mathrm{~cm}$. The energy and time resolutions for photon clusters are given by $\frac{\sigma_{E}}{E_{\gamma}}=\frac{0.057}{\sqrt{E_{\gamma}(\mathrm{GeV})}}$ and $\sigma_{t}=\frac{57 \mathrm{ps}}{\sqrt{E_{\gamma}(\mathrm{GeV})}} \oplus 100 \mathrm{ps}$.

The DC entrance wall composition is $750 \mu \mathrm{m}$ of carbon fiber and $150 \mu \mathrm{m}$ of aluminum foil. Dedicated GEANT Monte Carlo simulations of the KLOE apparatus show that out of the total number of kaons interacting in the DC entrance wall, about $81 \%$ are absorbed in the carbon fiber component and the remaining $19 \%$ in the aluminum foil. The KLOE DC is filled with a mixture of helium and isobutane $\left(90 \%\right.$ in volume ${ }^{4} \mathrm{He}$ and $10 \%$ in volume $\left.\mathrm{C}_{4} \mathrm{H}_{10}\right)$.

\section{Data samples}

The AMADEUS experiment is conceived to integrate the high acceptance and momentum resolution KLOE detector with the low momentum $\mathrm{K}^{-}$beam of the DAФNE collider in a future dedicated setup. As a first step, the data collected by the KLOE collaboration during the 2004/2005 data taking, for which the $d E / d x$ information of the reconstructed tracks is available $(d E / d x$ represents the truncated mean of the ADC collected counts due to the ionization in the DC gas), corresponding to $\sim 1.74$ $\mathrm{fb}^{-1}$, were analysed. The KLOE detector was used as an active target, the hadronic interaction of negative kaons with the materials of the apparatus being investigated. The topology of these data is shown in figure 1 , representing the radial position $\left(\rho_{\Lambda}\right)$ of the $\Lambda(1116)$ decay vertex (see Section 4). Four components are recognizable, from inside to outside we observe $K^{-}$absorptions in the DAФNE beryllium sphere $(\sim 5 \mathrm{~cm})$, the DAФNE aluminated beryllium pipe $(\sim 10 \mathrm{~cm})$, the KLOE DC entrance wall (aluminated carbon fiber $\sim 25 \mathrm{~cm}$ ) and the long tail originating from $K^{-}$interactions in the gas filling the KLOE DC $(25-200 \mathrm{~cm})$. Extremely rich experimental information is contained in this sample, with $K^{-}$hadronic interactions, both at-rest and in-flight [7], in a variety of light nuclear targets $\left(\mathrm{H},{ }^{4} \mathrm{He},{ }^{9} \mathrm{Be}\right.$ and $\left.{ }^{12} \mathrm{C}\right)$, which, of course, turned into a challenging analysis and interpretation efforts.

In order to increase the statistics and as an essential interpretation tool, a high purity carbon target (graphite) was realized in summer 2012 and installed inside the KLOE DC, between the beam pipe and the DC entrance wall.

The geometry of the target was optimized by means of a GEANT3 MC simulation. The final configuration was a three-sector half-cylinder $\left(\rho=0.95 \mathrm{~g} / \mathrm{cm}^{3}\right)$ supported by an aluminium frame, with a length of $600 \mathrm{~mm}$ and a mean radius of $24,7 \mathrm{~mm}$. The thickness varied from 4 to $6 \mathrm{~mm}$, in order to optimize the percentage of stopped kaons, taking into account the $\phi$ boost, derived from the crossing angle of the $\mathrm{e}^{+} \mathrm{e}^{-}$beams. The half-cylindrical configuration was chosen in order to take advantage of the $K^{+}$tagging in the opposite direction. The design of the realized target is shown in figure 2. We took data from 6 November to 14 December 2012, for a total integrated luminosity of $\sim 90 \mathrm{pb}^{-1}$. Up to now we analysed a sample of $37 \mathrm{pb}^{-1}$ reconstructed data. The topology of the reconstructed $\Lambda$ decay vertices radial position is shown in figure 3 , and as expected, the majority of kaons are stopped in the target.

\section{Particle Identification}

The investigation of the negatively charged kaons interactions in nuclear matter is performed through the reconstruction of hyperon-pion and hyperon-nucleon/nucleus correlated pairs productions, fol- 


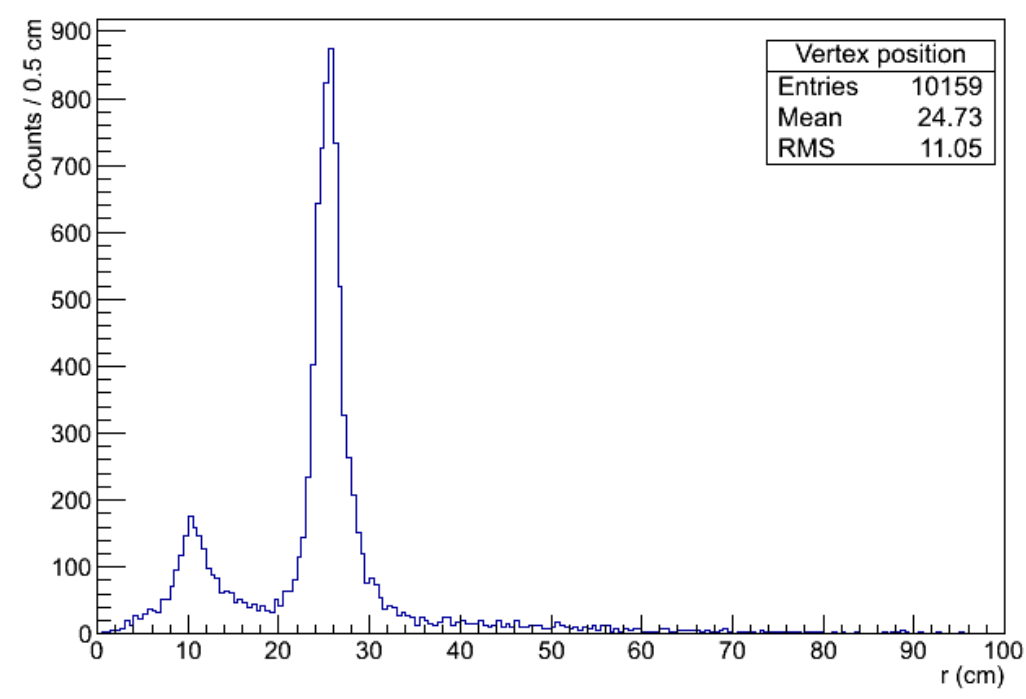

Figure 1. Radial position distribution $\rho_{\Lambda}$, of the $\Lambda$ decay vertex, for 2004-2005 KLOE collected data.

lowing the $\mathrm{K}^{-}$absorptions in $\mathrm{H},{ }^{4} \mathrm{He},{ }^{9} \mathrm{Be}$ and ${ }^{12} \mathrm{C}$. The $\Lambda(1116)$ identification proceeds through the reconstruction of the $\Lambda \rightarrow \mathrm{p}+\pi^{-}(\mathrm{BR}=63.9 \pm 0.5 \%)$ decay vertex. A spatial resolution below 1 $\mathrm{mm}$ is achieved for vertices found inside the DC volume (evaluated with Monte Carlo simulations). The obtained $M_{\mathrm{p} \pi^{-}}$invariant mass mean value is $1115.753 \pm 0.002 \mathrm{MeV} / \mathrm{c}^{2}$ (only statistical error is given, the systematics being under investigation), with a resolution of $\sigma=0.5 \mathrm{MeV} / \mathrm{c}^{2}$. The particle identification takes advantage of both $d E / d x$ information from the DC wires and the measurement of the energy released in the Calorimeter, as described in [1]. $\Sigma$ particles are identified through their decay into $\Lambda \gamma$ or $\mathrm{p} \pi$ as reported in $[7,8]$. The $\mathrm{K}^{-}$absorption vertex position, obtained exploiting the correlated production of the hyperon together with an extra-particle (pion, proton etc.) is then used to select the target. As an example, the obtained resolution on the radial coordinate $\left(\rho_{\Lambda \mathrm{p}}\right)$ for the $\Lambda \mathrm{p}$ vertex is $1.2 \mathrm{~mm}$. Cuts on the absorption vertex radial position were optimised, based on MC simulations and a study of the $\Lambda$ decay path, to select the targets with minimal contamination from other components. More details on the particle identification procedure can be found in [19]

\section{$5 \mathrm{~K}^{-}$absorption on two nucleons in the $\Sigma^{0} \mathrm{p}$ final state}

In [19], a high purity sample of $\Sigma^{0} \mathrm{p}$ events, from $\mathrm{K}^{-}$captures in the ${ }^{12} \mathrm{C}$, was reconstructed. $\Sigma^{0} \mathrm{p}$ is (together with $\Lambda \mathrm{p}$ ) an expected decay channel of the $\mathrm{ppK}^{-}$cluster, with the advantage to be free from the $\Sigma \mathrm{N} \rightarrow \Lambda \mathrm{N}^{\prime}$ conversion processes. The conversions strongly affect the uncorrelated $\Lambda \mathrm{p}$ production, thus distorting the observed spectra.

A simultaneus fit of the: $\Sigma^{0} \mathrm{p}$ invariant mass, the relative angle of the $\Sigma^{0}$ and proton in the laboratory system $\cos \left(\theta_{\Sigma^{0} \mathrm{p}}\right)$, the $\Sigma^{0}$ and the proton momenta was performed to the following simulated processes:

- $\mathrm{K}^{-} \mathrm{A} \rightarrow \Sigma^{0}-(\pi) \mathrm{p}_{\text {spec }}\left(\mathrm{A}^{\prime}\right)(1 \mathrm{NA})$,

- $\mathrm{K}^{-} \mathrm{pp} \rightarrow \Sigma^{0} \mathrm{p}$ (2NA), 


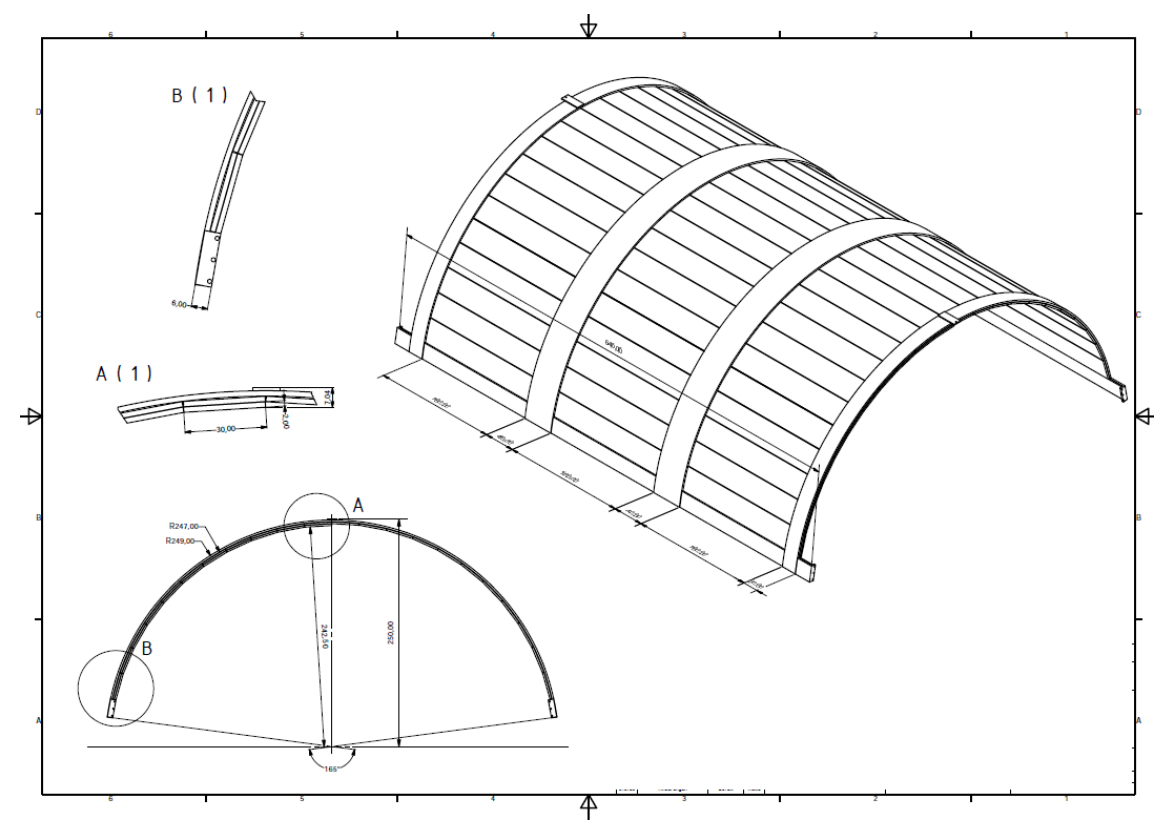

Figure 2. Design of the graphite target inserted between the beam pipe and the KLOE DC entrance wall.

- $\mathrm{K}^{-} \mathrm{ppn} \rightarrow \Sigma^{0} \mathrm{pn}(3 \mathrm{NA})$,

- $\mathrm{K}^{-} \mathrm{ppnn} \rightarrow \Sigma^{0} \mathrm{pnn}(4 \mathrm{NA})$.

Also Final State Interactions (FSI) of the $\Sigma^{0}$ and $\mathrm{p}$ emerging from a $\mathrm{K}^{-} \mathrm{pp}$ capture were taken into account.

The yield of the 2NA, when the produced $\Sigma^{0}$ and p particles are free form any FSI process, was measured for the first time, with good precision. The obtained results are summarized in Table 1. A second fit was carried out including a ppK ${ }^{-}$component, decaying into $\Sigma^{0} \mathrm{p}$. A systematic scan of possible binding energies and widths, varying within $15-75 \mathrm{MeV}$ and 30-70 MeV respectively, was performed. The best fit resulted in a binding energy of $45 \mathrm{MeV}$ and a width of $30 \mathrm{MeV}$. The resulting yield, normalised to the number of stopped $\mathrm{K}^{-}$, is $\mathrm{ppK}^{-} / \mathrm{K}_{\text {stop }}^{-}=\left(0.044 \pm 0.009 \mathrm{stat}_{-0.005}^{+0.004} \mathrm{syst}\right) \times 10^{-2}$. The significance of the bound state with respect to a statistical fluctuation was checked by means of an F-test and was found to be significant at the level of $1 \sigma$ only. Although the measured spectra are compatible with the hypothesis of a $\mathrm{ppK}^{-}$contribution, the significance of the result is not sufficient to claim the discovery of the state. We refer to [19] for the details of the analysis.

\section{$6 Y_{\pi}$ resonant and non resonant production and the shape of the $\Lambda(1405)$}

The position of the $\Lambda(1405)$ state is determined by the strength of the $\bar{K} N$ attractive interaction, thus influencing the possible formation of $\overline{\mathrm{K}}$ multi-N states formation. When extracting the $\Lambda(1405)$ shape from $\mathrm{K}^{-}$induced reactions in light nuclear targets (see for example [23]) the hyperon-pion spectroscopy is influenced by the energy threshold, imposed by the last nucleon binding energy. The $m_{\Sigma \pi}$ invariant mass threshold is about $1412 \mathrm{MeV}$ and $1416 \mathrm{MeV}$, for $\mathrm{K}^{-}$capture at-rest in ${ }^{4} \mathrm{He}$ and ${ }^{12} \mathrm{C}$ respectively, thus the $\mathrm{K}^{-}$absorption at-rest is not sensitive to the high mass pole predicted by chiral unitary models. The $\overline{\mathrm{K} N}$ sub-threshold region is accessible by exploiting $\mathrm{K}^{-} \mathrm{N}$ absorptions in-flight. 


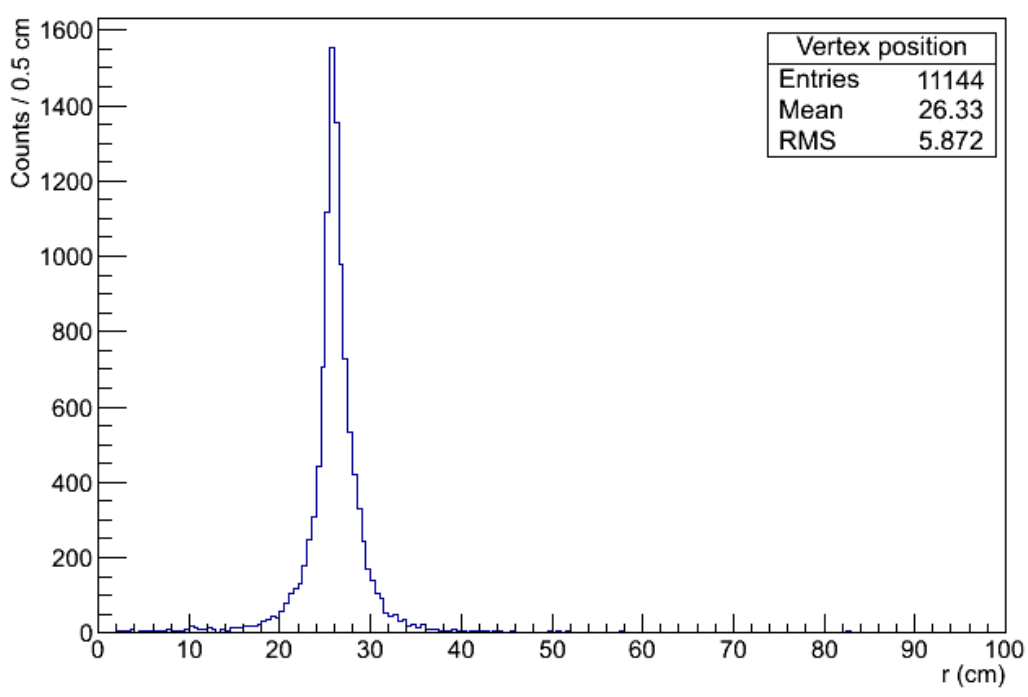

Figure 3. Radial position distribution of $\rho_{\Lambda}$ for carbon target events.

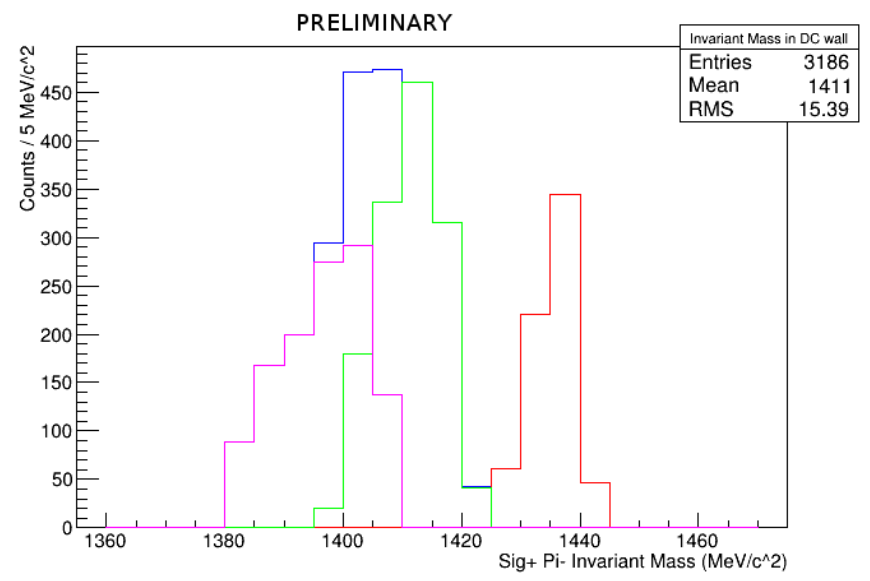

Figure 4. (Colour online.) $m_{\Sigma \pi}$ invariant mass distributions in-flight (green) and at-rest (violet) in ${ }^{12} \mathrm{C}$. Blue is the sum of green and violet curves. The red curve refers to $K^{-}$absorptions on Hydrogen

For a mean kaon momentum of $100 \mathrm{MeV} / \mathrm{c}$ the $m_{\Sigma \pi}$ threshold is shifted upwards by about $10 \mathrm{MeV}$. A second bias is represented by the non-resonant $\mathrm{K}^{-} \mathrm{N} \rightarrow \mathrm{Y} \pi$ formation, which gives rise to highly correlated hyperon-pion pairs production. The corresponding $m_{Y \pi}$ invariant masses spectra is narrow (of the order of $10 \mathrm{MeV}$ ) and peaked below the $\overline{\mathrm{K}} N$ threshold. The $\Lambda \pi$ and $\Sigma \pi$ non resonant transition amplitudes, for $\mathrm{K}^{-}$capture in light nuclear targets, was never measured. The $\Lambda$ and $\pi^{-}$kinematic dis- 


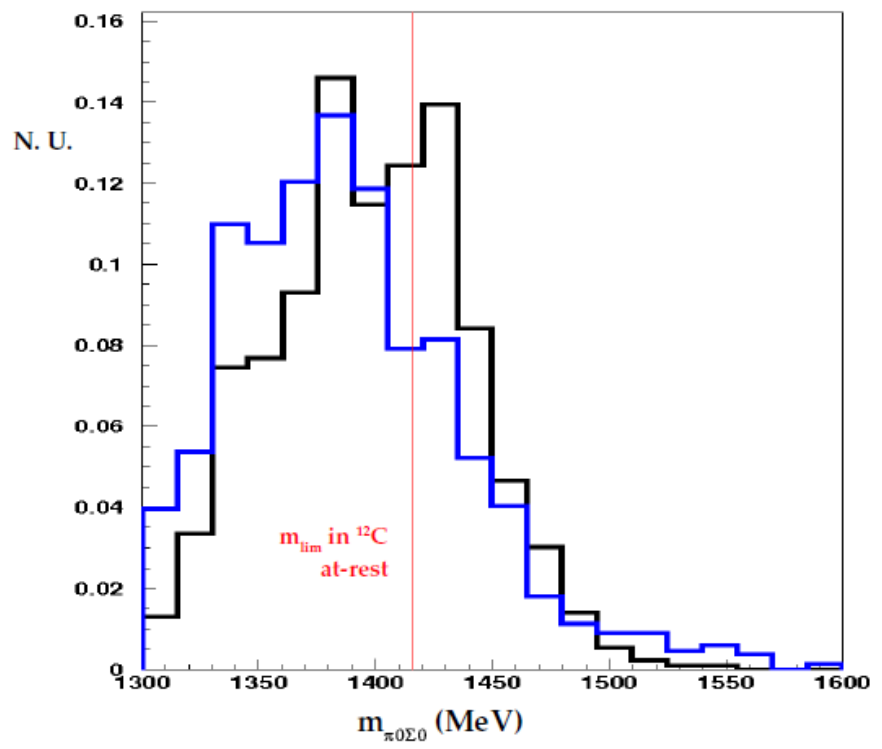

Figure 5. (Colour online.) $m_{\Sigma^{0} \pi^{0}}$ invariant mass distribution from $K^{-}$captures in the KLOE DC wall (black curve) and pure carbon graphite target (blue curve)

\begin{tabular}{cccc}
\hline Process & yield $/ \mathrm{K}_{\text {stop }}^{-} \times 10^{-2}$ & $\sigma_{\text {stat }} \times 10^{-2}$ & $\sigma_{\text {syst }} \times 10^{-2}$ \\
\hline 2NA-QF & 0.127 & \pm 0.019 & ${ }_{-0.008}^{+0.004}$ \\
2NA-FSI & 0.272 & \pm 0.028 & ${ }_{-0.023}^{+0.022}$ \\
Tot 2NA & 0.399 & \pm 0.033 & ${ }_{-0.032}^{+0.023}$ \\
3NA & 0.274 & \pm 0.069 & +0.044 \\
Tot 3 body & 0.546 & \pm 0.074 & -0.021 \\
4NA + bkg. & 0.773 & \pm 0.053 & -0.033 \\
\hline
\end{tabular}

Table 1. Production probability of the $\Sigma^{0} \mathrm{p}$ final state for different intermediate processes normalised to the number of stopped $\mathrm{K}^{-}$in the DC wall. The statistical and systematic errors are shown as well [19].

tributions for $\mathrm{K}^{-}$captures in ${ }^{4} \mathrm{He}$, both at-rest and in-flight, were calculated in [22]. The momentum probability distribution functions of the emerging hyperon pion pairs, following $\mathrm{K}^{-} \mathrm{n}$ absorptions, are expressed in terms of the $\mathrm{K}^{-} \mathrm{n}$ transition amplitudes: the isospin $I=1 \mathrm{~S}$-wave non- resonant amplitude $\left(\left|f^{\mathrm{nr}}\right|\right)$ and the resonant $I=1 \mathrm{P}$-wave amplitude, dominated by the $\Sigma^{-}(1385)$. Since the resonant amplitude is well known from direct experiments, the measured total momentum distributions can be used to extract the non-resonant $\left|f^{\mathrm{nr}}\right|$ amplitude module below the $\overline{\mathrm{K}} \mathrm{N}$ threshold. The goal of the ongoing analyses is to measure the contributions and the shapes of the non resonant $\Lambda \pi$ and $\Sigma \pi$ productions. The knowledge of the $(\Sigma \pi)^{0}$ isospin $I=0$ non-resonant transition amplitude will allow to disentangle the resonant $\Lambda(1405)$ shape. Preliminary $\Sigma^{+} \pi^{-}$invariant mass spectra, from $\mathrm{K}^{-}$captures in the wall of the KLOE DC, not background subtracted nor acceptance corrected, are shown in Fig. 4. The red curve refers to $\mathrm{K}^{-}$absorptions on Hydrogen, green and violet distributions refer to $\mathrm{K}^{-}$ 
captures in-flight and at-rest in ${ }^{12} \mathrm{C}$ respectively, the blue line is the sum of green and violet distributions. The red distribution reflects the non-resonant $\mathrm{K}^{-} \mathrm{H}$ absorption in-flight, which corresponds to a narrow invariant mass shape peaked below $m_{K}+m_{p}+\left\langle p_{K}^{2}\right\rangle / 2 m_{K}$, the third term represents the mean kinetic energy of the non-relativistic kaons. In figure 5 (black distribution) the $\Sigma^{0} \pi^{0}$ invariant mass spectrum from $\mathrm{K}^{-}$captures in the KLOE DC wall is shown [25]. The reduced resolution, due to the reconstruction of the three photons clusters, does not allow in this case to separate the two processes of at-rest and in-flight capture, moreover the absorbing target $\left(\mathrm{H}\right.$ or $\left.{ }^{12} \mathrm{C}\right)$ can not be distinguished in this case. The $m_{\Sigma 0 \pi 0}$ spectrum can be compared with the corresponding distribution from $\mathrm{K}^{-}$absorptions in a pure carbon target (fig. 5 blue distribution), sample which consists of $\mathrm{K}^{-}$captures at-rest. In fig. 5 a red line indicates the energy threshold corresponding to $\mathrm{K}^{-}$absorption in ${ }^{12} \mathrm{C}$ at-rest. In order to compare the two distributions plotted in fig. 5 the two curves are normalised to unity. A rich sample of in-flight $\mathrm{K}^{-12} \mathrm{C}$ captures can be easily identified. A spectroscopic study of the kinematic region ranging between the at-rest energy threshold, and the $\overline{\mathrm{K}} \mathrm{N}$ threshold, opened by the low momentum in-flight capture process, will allow to clarify the nature of the high mass $\Lambda(1405)$ pole.

\section{Conclusions and perspectives}

In this work a broad research program of low-energy $\mathrm{K}^{-}$induced reactions on light nuclear targets is presented. The $\overline{\mathrm{K}} \mathrm{N}$ interaction in nuclear matter is investigated through the study of the hyperon resonances properties below the $\overline{\mathrm{K}} \mathrm{N}$ threshold, and the characterization of $\mathrm{K}^{-}$-multi-nucleon captures processes. The latter is found to strongly impact on the $\mathrm{K}^{-}$-multi-nucleon bound state search, as the $\mathrm{K}^{-} \mathrm{pp}$ (2NA) absorption overlaps with the bound state in the phase space region where it is expected.

In the search for the $\mathrm{K}^{-}$pp bound state, and to extract the $\mathrm{K}^{-}$-multi-nucleon absorption yields, we faced the problem of disentangling the elastic and inelastic final state interactions of the hyperons with the residual nucleons. The former process is of particular interest for the measurement of the hyperonnucleon (multi-nucleon) cross sections, for which the available experimental information is extremely scarce. Moreover, the hyperon-nucleon (multi-nucleon) interaction potentials are fundamental inputs in the determination of the equation of state for the neutron stars, whose structure is strongly debated, following the measurement of two neutron stars exceeding $2 \mathrm{M} \odot$ [26, 27]. Experimental constrains from the hyperon-nucleon scattering processes are mandatory to guide the theory.

Presently a feasibility study [28,29] is ongoing for the realization of a dedicated AMADEUS experimental setup, in order to deepen and extend the low energy anti-kaon nuclei interaction studies and obtain fundamental input for the study of QCD with strangeness and of neutron stars.

\section{Acknowledgement}

We acknowledge the KLOE Collaboration for their support and for having provided us the data and the tools to perform the analysis presented in this paper.

\section{References}

[1] C. Curceanu, K. Piscicchia et al., Acta Phys. Polon. B46 1, 203-215, (2015).

[2] R. Baldini et al., Proposal for a Phi-Factory, report LNF-90/031(R) (1990).

[3] F. Bossi, E. De Lucia, J. Lee-Franzini, S. Miscetti, M. Palutan and KLOE coll., Riv. Nuovo Cim. 31 (2008) 531-623

[4] C. Patrignani et al. (Particle Data Group), Chin. Phys. C, 40, 100001 (2016).

[5] T. Hyodo, D. Jido, Prog. Part. Nucl. Phys. 67 (2012) 55. 
[6] Phys. Lett. B 500 (2001),

Phys. Rev. C 66 (2002),

Nucl. Phys. A 725 (2003),

Nucl. Phys. A881, 98 (2012)

[7] K. Piscicchia et al., PoS Bormio2013 034 (2013)

[8] A. Scordo et al., PoS Bormio2014 039 (2014)

[9] T. Yamazaki, et al., Phys. Rev. C 76045201 (2007)

A. Doté, et al., Phys. Rev. C 79014003 (2009)

S. Wycech, et al., Phys. Rev. C 79014001 (2009)

N. Barnea, et al., Phys. Lett. B 712132 (2012)

N.V. Shevchenko, et al., Phys. Rev. Lett. 98082301 (2007)

Y. Ikeda, et al., Phys. Rev. C 79035201 (2009)

E. Oset, et al., Nucl. Phys. A 881127 (2012)

[10] G. Agakishiev, et al., HADES Coll., Phys. Lett. B 742242 (2015)

[11] M. Agnello, et al. (FINUDA Coll.), Phys. Rev. Lett. 94212303 (2005)

[12] T. Yamazaki, et al., Phys. Rev. Lett. 104132502 (2010)

[13] Y. Ichikawa, et al., Prog. Theor. Exp. Phys. $021 D 01$ (2015)

[14] A. O. Tokiyasu et al., Phys.Lett. B 728, 616 (2014)

[15] L. Fabbietti et al., Nucl. Phys. A 914, 60 (2013)

[16] T. Hashimoto et al., Prog. Theor. Exp. Phys. 061D01 (2015)

[17] M. Adinolfi et al., [KLOE Collaboration], Nucl. Inst. Meth. A 488, (2002) 51.

[18] M. Adinolfi et al. [KLOE Collaboration], Nucl. Inst. Meth. A 482, (2002) 368.

[19] O. Vazquez Doce et al., Phys. Lett. B 758, 134-139 (2016)

[20] R. Roosen, J. H. Wickens, Il Nuovo Cim. A Series 11, 66101 (1981)

[21] FINUDA collaboration, Phys. Lett. B, 669229 (2008)

[22] K. Piscicchia, S. Wycech, C. Curceanu, Nucl. Phys. A 954, 75-93 (2016)

[23] J. Esmaili et al., Phys. Lett. B 686 (2010) 23-28

[24] FINUDA collaboration, Phys. Lett. B 704, 474-480 (2011)

[25] K. Piscicchia, PhD theis (2013), Lambda(1405) measurement through the decay to $\Sigma^{0} \pi^{0}$, resulting from $\mathrm{K}^{-}$meson absorption on ${ }^{4} \mathrm{He}$ and ${ }^{12} \mathrm{C}$, with the $\mathrm{KLOE}$ detector, http://www.infn.it/thesis/thesis_dettaglio.php?tid=7097

[26] P. B. Demorest, T. Pennucci, S. M. Ransom, M. S. E. Roberts \& J. W. T. Hessels, Nature (London) 467, (2011)

[27] D. J. Nice, E. M. Splaver, I. H. Stairs, O. Loehmer, and A. Jessner, Astrophys. J. 634, 1242 (2005).

[28] M. Bazzi, et al., Nuclear Instruments and Methods in Physics Research A 671 (2012) 125-128

[29] M. Bazzi et al., Journal of Instrumentation 8(05) January 2013 